\title{
High Conversion of Palm Olein to Ethyl Esters Using a Strong Anion Exchange Resin: Study of the Operational Parameters
}

\author{
Isabel J. Bejarano-Alva, ${ }^{a}$ Gisele A. M. Hirata, ${ }^{b}$ Klicia A. Sampaio, ${ }^{a}$ \\ Eduardo A. C. Batista ${ }^{a}$ and Antonio J. A. Meirelles ${ }^{\oplus} * a$ \\ ${ }^{a}$ Faculdade de Engenharia de Alimentos, Universidade Estadual de Campinas (Unicamp), \\ 13083-862 Campinas-SP, Brazil \\ ${ }^{b}$ Departamento de Engenharia Química, Universidade Federal de São Paulo (Unifesp), \\ Campus Diadema, 09913-030 Diadema-SP, Brazil
}

\begin{abstract}
Biodiesel, known as a mixture of fatty acid ethyl/methyl esters, is seen as an alternative, ecofriendly, biodegradable and renewable non-fossil fuel. The use of heterogeneous catalysts for biodiesel synthesis can solve several problems associated with the homogeneous alkaline catalyzedtransesterification. Therefore, this work reports the evaluation of the commercial resin Amberlyst $\mathrm{A} 26 \mathrm{OH}$, a strong anion exchange resin, as a heterogeneous catalyst for the batch transesterification of refined palm olein with ethanol. It was studied the effects of the main operational parameters, considering the molar ratio of the reaction mixture (MRRM), namely the molar ratio of ethanol to olein taking into account only the ethanol added to the reaction system, and the total molar ratio (TMR), in this case considering also the amount of ethanol carried by the resin after its pretreatment. It was determined an optimal range of operational conditions by response surface methodology, guaranteeing conversion to ethyl esters higher than $96 \%$ with a catalyst amount corresponding to a range from 10.4 to $11.4 \%$ of the oil quantity, a temperature within the range of 55 to $60{ }^{\circ} \mathrm{C}$ and a MRRM within the range from 3.5:1 to $6.0: 1$.
\end{abstract}

Keywords: strong anion exchange resin, transesterification, biodiesel, heterogeneous catalysis, rotatable central composite design, palm olein

\section{Introduction}

Approximately $80 \%$ of the global energy supply comes from fossil fuels. Awareness about fossil fuels' contribution to global warming as well as concerns about the planet's sustainability stimulate the search for solutions such as alternative energy sources. ${ }^{1,2}$

Biodiesel has advantages like easy availability from common biomass sources, contribution to the reduction of greenhouse gas (GHG) emissions, environmental friendliness potential and fuel security for countries with little or no oil reserves of their own. ${ }^{3}$ Biodiesel production may be performed by transesterification of fats and oils (acylglycerols) using an appropriate catalyst for speeding up the exchange of the glycerol moiety by the alkoxy moieties of short chain alcohols. ${ }^{4-6}$

Although alkaline homogeneous catalysis is the most often used technique for transesterification due to: (i) its

*e-mail: tomze@unicamp.br relatively low cost compared to heterogeneous and enzymatic catalysts; (ii) the easy availability of alkaline catalysts in the market, and (iii) its ability to accelerate transesterification effectively under mild reaction conditions, heterogeneous catalysis has various advantages. ${ }^{7,8}$ Unlike homogeneous catalysis, heterogeneous catalysts do not generate soaps, enable simpler product and catalyst separation, decreasing the need for downstream purification, and allow waste treatment in a more environmental friendly way. Furthermore, heterogeneous catalysis facilitates catalyst reuse and the implementation of continuous processing. ${ }^{9,10}$ As disadvantages one can mention the higher costs and special temperature and pressure requirements. ${ }^{11,12}$

A strong anion exchange resin, with its quaternary ammonium as fixed-ion and a hydroxyl as counterion (see Figure 1), can be used as heterogeneous catalysts on the transesterification reaction. At the start of the reaction mechanism, the Lewis' alkaline site of the catalyst $\left(\mathrm{OH}^{-}\right)$ interacts with an ethanol molecule, producing an ethoxyl and protonating the catalyst. This acid-base interaction gives rise 
to a nucleophilic attack on the carbon of the triacylglycerol carbonyl group, carried out by the oxygen electron pair located in the ethanol hydroxyl group, which produces a tetrahedral intermediate. In its turn, this intermediate generates a diacylglycerol molecule and an alkoxide ion, which finally deprotonates the catalyst to produce the ethyl ester molecule. The diacylglycerol produced can react with another ethoxyl, starting a second catalytic cycle that produces a monoacylglycerol and another ester molecule. At the end, the sequence of three consecutive and reversible transesterification reactions generates three molecules of ethyl esters and one glycerol molecule. . $^{13,14}$

In the batch production of fatty esters by heterogeneous catalysis, the parameters usually investigated are: reaction temperature, molar ratio of ethanol to oil, stirring intensity, proportion catalyst:oil and solid phase catalytic activity.

In the case of ion exchange resins some peculiarities can also influence their activity either as an adsorbent or as a catalyst. ${ }^{15}$ Deboni et al. ${ }^{16}$ emphasized these aspects in their transesterification studies. Ion exchange resins have a highly porous structure, able to retain a large amount of liquid phase, especially polar compounds, such as water molecules. Their use as catalysts may require pretreatment steps for withdrawing the excessive amounts of water that could impair their activity in an organic media. If this pretreatment is performed with the same solvent used in transesterification, the consequence would be a change in the molar ratio of the reagents added to the reactor. Furthermore, the reaction development occurs simultaneously with the mass transfer of components between the liquid phase contained within the resin porous structure and the bulk phase around its beads, so that components eventually adsorbed to the resin internal surface, such as glycerol molecules, may affect the access to its active sites. Despite their possible influence on the resin catalytic activity, such factors were not considered in prior investigations.

In summary, although there have been previous studies on the transesterification process using ion exchange resins as a heterogeneous catalyst, some of them with very promising results, ${ }^{11,16-23}$ these studies have not reported the optimal parameters for the ethanolysis reaction or took into account the influence of the resin pretreatment on the conversion results. The main contribution of the present work is the optimization of operational parameters, such as temperature, catalyst amount and molar ratio, in the ethanolysis of vegetable oils using a strong anion exchange resin (Amberlyst $\mathrm{A} 26 \mathrm{OH}$ ). In addition, the work also addresses the possible influence of the resin pretreatment, as well as the alcohol content retained inside its pores, upon the transesterification process. Ethanol as the alcoholic source replacing methanol has the advantage of a lower environmental impact, due to its production based on the biotechnological route. The source of fatty compounds was palm olein, a fraction of palm oil that generates a biofuel also adequate for cold climates and has the advantage of being based on the most productive agricultural source of fatty compounds.

\section{Experimental}

\section{Materials}

Refined palm olein was kindly supplied by Agropalma S/A (Belém, Brazil). Its initial acidity was

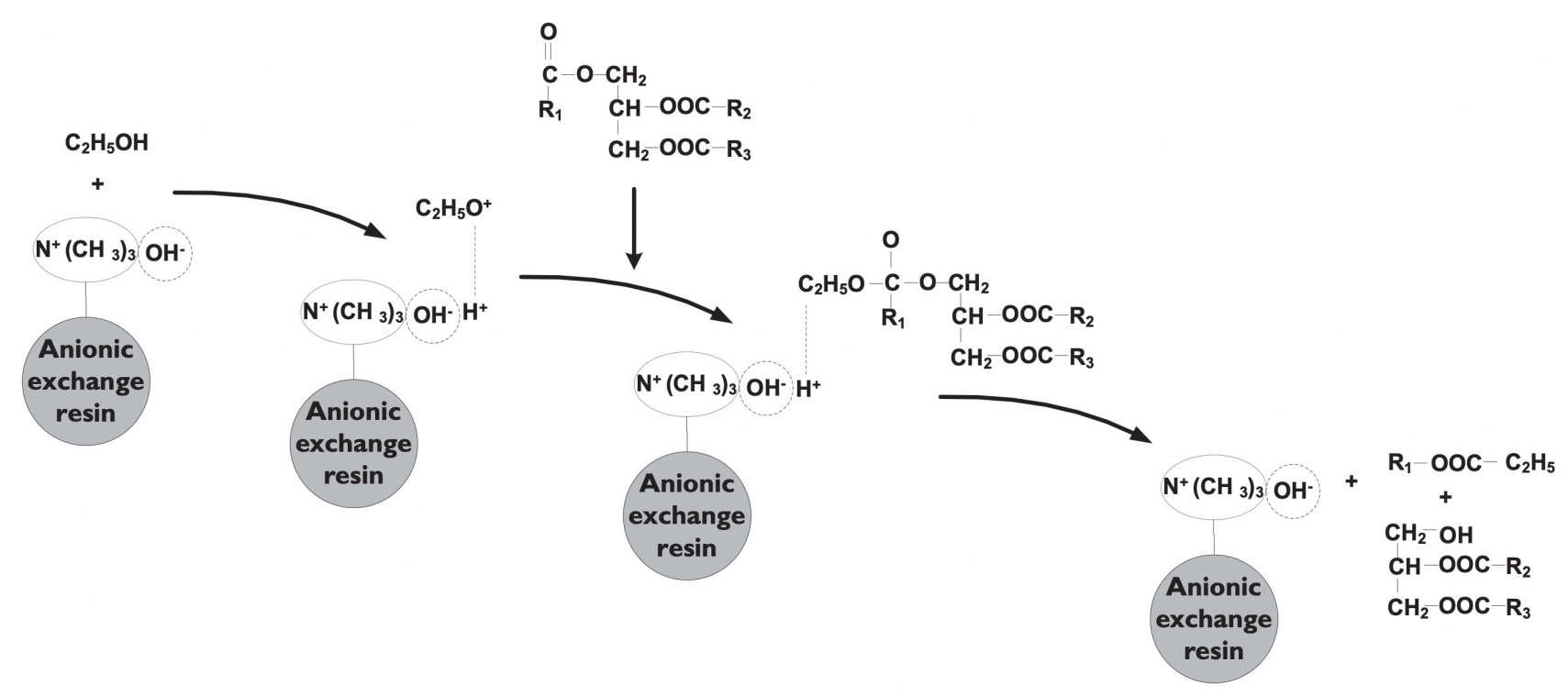

Figure 1. Transesterification mechanism using a strong anion exchange resin. ${ }^{13,14}$ 
$0.07 \%$ (expressed as oleic acid). Ethanol used as reactant $(\geq 99.9 \%$ ) was purchased from Merck (Darmstadt, Germany). Ethanol used for the resin pretreatment was 99.5\% pure, and obtained from Dinâmica (São Paulo, Brazil). Toluene was acquired from Sigma-Aldrich (Saint Louis, USA). The strong anion exchange resin Amberlyst A26OH was purchased from Dow Brazil S/A (São Paulo, Brazil). ${ }^{24}$ According to the manufacturer, Amberlyst $\mathrm{A} 26 \mathrm{OH}$ has the following characteristics: spherical beads with mean size within the range $0.56-0.70 \mathrm{~mm}$, average pore diameter $290 \AA$, concentration of active site $\geq 80$ equiv. $\mathrm{L}^{-1}$, moisture holding capacity $66-75 \%$ (OH form). The pretreatment of the resin for withdrawing the excessive amounts of water inside was performed as recommended by Deboni et al. ${ }^{16}$ The resin was washed with 5 volumes of ethanol (in relation to the resin volume) in a fixed bed, using an ethanol flow rate of 2 bed volumes $\mathrm{h}^{-1}$. After pretreatment the resin was stored immersed in ethanol and, before its use in the experimental runs, the resin beads were separated by filtration. The amount of ethanol available inside the resin due to its pretreatment was determined on triplicate by the gravimetric method. About $1 \mathrm{~g}$ of sample was dried in an air circulation oven (Marconi-model MA035) at $110^{\circ} \mathrm{C}$ until weight remained constant.

\section{Palm olein characterization}

In order to determine the fatty acid profile, refined palm olein was converted to fatty acid methyl esters as reported by Hartman and Lago. ${ }^{25}$ The fatty compounds were analyzed by gas chromatography to determine the fatty acid composition according to the AOCS official method Ce 1-62. ${ }^{26}$ The refined olein contained 35.94 mass $\%$ of hexadecanoic acid, 46.65 mass $\%$ of cis-9-octadecenoic acid and 10.21 mass $\%$ of cis-9,cis-12-octadecadienoic acid (Table 1).

Table 1. Fatty acid composition of refined palm olein

\begin{tabular}{lccc}
\hline Fatty acid & Symbol & Cx:y & $\begin{array}{c}\text { Refined palm } \\
\text { olein / mass\% }\end{array}$ \\
\hline Dodecanoic & $\mathrm{L}$ & $12: 0$ & 1.30 \\
Tetradecanoic & $\mathrm{M}$ & $14: 0$ & 0.70 \\
Hexadecanoic & $\mathrm{P}$ & $16: 0$ & 35.94 \\
9-Hexadecenoic & $\mathrm{Po}$ & $16: 1$ & 0.10 \\
Octadecanoic & $\mathrm{S}$ & $18: 0$ & 4.40 \\
cis-9-Octadecenoic & $\mathrm{O}$ & $18: 1$ & 46.65 \\
cis-9,cis-12-Octadecadienoic & $\mathrm{Li}$ & $18: 2$ & 10.21 \\
All-trans-9,12,15-octadecatrienoic & $\mathrm{Ln}$ & $18: 3$ & 0.30 \\
Icosanoic & $\mathrm{A}$ & $20: 0$ & 0.40 \\
\hline
\end{tabular}

${ }^{\mathrm{a}} \mathrm{Cx}: \mathrm{y}$ : $\mathrm{x}$ is the number of carbons and $\mathrm{y}$ is the number of double bonds.
A PerkinElmer gas chromatographic system, Clarus 600, equipped with a flame ionization detector (FID) and a PerkinElmer Elite-225 capillary column (crossbond, 50\% cyanopropylmethyl $50 \%$ phenylmethyl polysiloxane), length of $30 \mathrm{~m}$, internal diameter of $0.25 \mathrm{~mm}$ and film thickness of $0.25 \mathrm{~mm}$, was used to analyze the samples. The following operating conditions were selected: helium (carrier gas) at a flow rate of $1.78 \mathrm{~mL} \mathrm{m^{-1 }}$, FID temperature of $250{ }^{\circ} \mathrm{C}$, injector at $250{ }^{\circ} \mathrm{C}$, injection volume of $1 \mu \mathrm{L}$, column temperature ramp from 50 to $250^{\circ} \mathrm{C}$ at $10^{\circ} \mathrm{C} \mathrm{min}^{-1}$. Retention times and peak areas were evaluated via TotalChrom software (version 6.3.2, PerkinElmer, USA).

From the fatty acid composition, the probable triacylglycerol composition was calculated using the statistical algorithm suggested by Antoniosi Filho et al. ${ }^{27}$ These authors compared experimental data obtained by gas chromatography for several vegetable oils and the results acquired by the statistical method showed a good correlation between the gas chromatographic data and the statistical data (Table 2). The algorithm proposed by Antoniosi Filho et al. ${ }^{27}$ is based on the theory of 1,3-random-2-random distribution, which states that the fatty acids are statistically distributed at random among the three positions of the glycerol moiety, but with the C-2 hydroxyl group being preferentially acylated by fatty acids with the highest degree of unsaturation. For calculations of the probable triacylglycerol composition, two more aspects were considered: the composition of trans isomers were added to its respective $c i s$ isomer and triacylglycerols with less than $0.5 \%$ were ignored.

The olein composition in terms of acylglycerol classes (tri-, di- and monoacylglycerols) was analyzed by liquid chromatography according to the methodology indicated in the "Analytical methods" sub-section. In terms of these classes the olein total composition was 94.73 mass\% of triacylglycerols and 5.27 mass\% of diacylglycerols. No monoacylglycerol class was identified according to the method's limit of detection. The composition in diacylglycerols were estimated considering the probability of the partial rupture of triacylglycerols without preference for specific ester bonds (Table 2).

The olein acidity was determined by titration according to IUPAC methodology $2.2012^{28}$ using an automatic titrator model 808 Titrando (Metrohm, Switzerland).

\section{Transesterification step}

The transesterification reaction was carried out by heterogeneous catalysis in a glass reactor placed on a magnetic stirrer plate, stirred at $400 \mathrm{rpm}$ with a cylindrical magnetic bar suspended in a metal support. 
Table 2. Probable tri- and diacylglycerol compositions of the refined palm olein

\begin{tabular}{|c|c|c|c|c|c|}
\hline \multirow{2}{*}{ Main $\mathrm{TAG}^{\mathrm{a}}$} & \multicolumn{2}{|c|}{ Molar mass } & \multirow{2}{*}{$\mathrm{DAG}^{\mathrm{b}}$} & \multicolumn{2}{|c|}{ Molar mass } \\
\hline & $\left(\mathrm{g} \mathrm{moL}^{-1}\right)$ & $\%$ molar & & $\left(\mathrm{g} \mathrm{moL}^{-1}\right)$ & $\%$ molar \\
\hline LOP & 777.26 & 0.65 & OO- & 620.98 & 18.52 \\
\hline MOP & 805.32 & 1.63 & OLi- & 618.96 & 7.16 \\
\hline POP & 833.37 & 34.76 & PO- & 594.94 & 42.41 \\
\hline POS & 861.42 & 7.51 & SO- & 622.99 & 3.9 \\
\hline LLiP & 775.25 & 0 & PLi- & 592.92 & 9.25 \\
\hline OOL & 803.3 & 0 & OA- & 651.05 & 0.27 \\
\hline POA & 889.48 & 0 & LiLi- & 616.95 & 0.84 \\
\hline SOS & 889.48 & 0.63 & LP- & 512.80 & 1.01 \\
\hline MLiP & 803.3 & 0.54 & LO- & 538.83 & 1.98 \\
\hline PLiP & 831.35 & 7.17 & LLi- & 536.82 & 0.36 \\
\hline OOP & 859.41 & 26.12 & MP- & 540.85 & 0.5 \\
\hline OOS & 887.46 & 2.8 & MO- & 566.89 & 0.5 \\
\hline LOLi & 801.28 & 0 & PP- & 568.90 & 11.07 \\
\hline POLi & 857.39 & 9.43 & PS- & 596.96 & 1.96 \\
\hline $\mathrm{OOO}$ & 885.44 & 5.44 & PA- & 625.01 & 0.27 \\
\hline LiLiP & 855.38 & 1.1 & & & \\
\hline OOLi & 883.43 & 1.68 & & & \\
\hline $\mathrm{LiLiO}$ & 881.41 & 0.54 & & & \\
\hline
\end{tabular}

a Groups with a total triacylglycerol (TAG) concentration lower than $0.5 \%$ were ignored; bAG: diacylglycerol. L: dodecanoic; M: tetradecanoic; P: hexadecanoic; Po: 9-hexadecenoic; S: octadecanoic; O: cis-9-octadecenoic; Li: cis-9,cis-12-octadecadienoic; Ln: all-trans-9,12,15-octadecatrienoic; A: icosanoic.

It was important to suspend the magnetic bar since the stirring bar lying on the reactor bottom tends to break the resin beads. A thermometer was placed inside the reactor to monitor the reaction temperature. Each component was weighed on a semi analytical balance with a precision of $0.01 \mathrm{~g}$ (Gehaka BK-500, Brazil).

Afterwards, known quantities of the previously pretreated resin (Amberlyst $\mathrm{A} 26 \mathrm{OH}$ ) and anhydrous ethanol were placed together into the reactor. The solution was kept under constant stirring during $15 \mathrm{~min}$, and after that olein was added. The temperature of the reaction was kept constant during the whole experiment, by the use of a thermostatic water bath. Samples were withdrawn in specified time intervals to determine the concentration of fatty acid ethyl esters (FAEE) along the transesterification reaction.

Olein conversion to FAEE was calculated by equation 1 , which relates the amount of ethyl esters produced with the moles of oil, in fact the acylglycerols, used during the transesterification.

Conversion (\%) $=\left[1-\frac{\left(3 \times \mathrm{N}_{\mathrm{TAG}, \mathrm{f}}+2 \times \mathrm{N}_{\mathrm{DAG}, \mathrm{f}}+1 \times \mathrm{N}_{\mathrm{MAG}, \mathrm{f}}\right)}{\left(3 \times \mathrm{N}_{\mathrm{TAG}, \mathrm{i}}+2 \times \mathrm{N}_{\mathrm{DAG}, \mathrm{i}}+1 \times \mathrm{N}_{\mathrm{MAG}, \mathrm{i}}\right)}\right] \times 100$

where $\mathrm{N}_{\mathrm{TAG}, \mathrm{i}}, \mathrm{N}_{\mathrm{DAG}, \mathrm{i}}, \mathrm{N}_{\mathrm{MAG}, \mathrm{i}}$ are initial moles of tri-, diand monoacylglycerols respectively, and $\mathrm{N}_{\mathrm{TAG}, \mathrm{f}}, \mathrm{N}_{\mathrm{DAG}, \mathrm{f}}$,
$\mathrm{N}_{\text {MAG,f }}$ are final moles of tri-, di- and monoacylglycerols, respectively.

\section{Optimization strategy}

The investigation of the parameters' influence on the transesterification reaction was performed in three successive steps. The experiments initially comprised a screening carried out in order to understand the effect of time on the olein conversion. Afterwards a first rotational central composite design (F-RCCD) was established maintaining the selected time obtained in the preliminary study. In this experimental design (F-RCCD), the molar ratio ethanol:olein was calculated taking into account only the molar ratio of the reaction mixture (MRRM). In the last step, a second rotational central composite design (S-RCCD) was performed with total molar ratio (TMR).

\section{Screening for the reaction time}

For these trials we selected two catalyst concentrations (6 and 10 mass\%). During these screening trials, the other transesterification parameters, such as temperature $\left(45^{\circ} \mathrm{C}\right)$, stirring speed (400 rpm), and MRRM of 9:1, were maintained constant. In this set of experiments, the maximum reaction time was fixed at $12 \mathrm{~h}$. 
Table 3. First experimental matrix for the central composite design in transesterification using Amberlyst A26OH

\begin{tabular}{|c|c|c|c|c|c|c|c|}
\hline Run & $\mathrm{X}_{1}$ & $\mathrm{X}_{2}$ & $\mathrm{X}_{3}$ & Temperature $/{ }^{\circ} \mathrm{C}$ & $\begin{array}{c}\text { Catalyst amount / } \\
\text { mass\% }\end{array}$ & $\begin{array}{l}\text { Molar ratio of the } \\
\text { mixture }\end{array}$ & Conversion / \% \\
\hline 1 & -1 & -1 & -1 & 45 & 6 & $4.8: 1$ & 73.1 \\
\hline 2 & 1 & -1 & -1 & 65 & 6 & $4.8: 1$ & 79.5 \\
\hline 3 & -1 & 1 & -1 & 45 & 10 & $4.8: 1$ & 92.4 \\
\hline 4 & 1 & 1 & -1 & 65 & 10 & $4.8: 1$ & 96.8 \\
\hline 5 & -1 & -1 & 1 & 45 & 6 & $8.8: 1$ & 66.6 \\
\hline 6 & 1 & -1 & 1 & 65 & 6 & $8.8: 1$ & 84.6 \\
\hline 7 & -1 & 1 & 1 & 45 & 10 & $8.8: 1$ & 90.6 \\
\hline 8 & 1 & 1 & 1 & 65 & 10 & $8.8: 1$ & 96.8 \\
\hline 9 & -1.68 & 0 & 0 & 38.2 & 8 & $6.8: 1$ & 76.8 \\
\hline 10 & 1.68 & 0 & 0 & 71.8 & 8 & $6.8: 1$ & 88.8 \\
\hline 11 & 0 & -1.68 & 0 & 55 & 4.64 & $6.8: 1$ & 64.9 \\
\hline 12 & 0 & 1.68 & 0 & 55 & 11.36 & $6.8: 1$ & 97.0 \\
\hline 13 & 0 & 0 & -1.68 & 55 & 8 & $3.44: 1$ & 86.1 \\
\hline 14 & 0 & 0 & 1.68 & 55 & 8 & $10.16: 1$ & 89.2 \\
\hline $15(\mathrm{C})$ & 0 & 0 & 0 & 55 & 8 & $6.8: 1$ & 88.3 \\
\hline $16(\mathrm{C})$ & 0 & 0 & 0 & 55 & 8 & $6.8: 1$ & 92.1 \\
\hline $17(\mathrm{C})$ & 0 & 0 & 0 & 55 & 8 & $6.8: 1$ & 88.5 \\
\hline
\end{tabular}

Coded variables: $\mathrm{X}_{1}$ (temperature); $\mathrm{X}_{2}$ (catalyst amount) and $\mathrm{X}_{3}$ (molar ratio of the mixture, MRRM). C: central point.

\section{First rotatable central composite design (F-RCCD)}

For the first statistical design, the parameters temperature $\left(\mathrm{X}_{1}\right)$, amount of catalyst $\left(\mathrm{X}_{2}\right)$ and molar ratio of the reaction mixture $\left(\mathrm{X}_{3}\right)$ were chosen as independent variables, and the conversion value (equation 1 ) was the dependent one.

The experiments were performed using a rotatable central composite design. The reaction time was constant for all experiments and its value selected according to the screening study. Each factor had five different levels: two combining factorial points $( \pm 1)$, two axial points $( \pm 1.68)$ and a central point (0) (Table 3). The experimental design applied had 14 experiments and 3 central points (Table 3).

\section{Second rotatable central composite design (S-RCCD)}

For the second statistical design, a minimum temperature was selected that allowed the highest conversion in the F-RCCD. The variables amount of catalyst $\left(\mathrm{Y}_{1}\right)$ and total molar ratio $\left(\mathrm{Y}_{2}\right)$ were investigated, considering in the last factor $\left(\mathrm{Y}_{2}\right)$ also the amount of ethanol carried by the resin after its pretreatment (approximately 80 mass $\%$ of the pretreated resin). Each factor had five different levels: two combining factorial points $( \pm 1)$, two axial points $( \pm 1.41)$ and a central point ( 0$)$. The experimental design applied had 8 experiments and 3 central points (Table 4).

\section{Statistical analysis}

The experimental data were analyzed using the software Statistica v13.1 (Statsoft, 2015) ${ }^{29}$ to establish the effects of the process variables on the response of interest. The
Table 4. Second experimental matrix for the central composite design

\begin{tabular}{lccccc}
\hline Run & $\mathrm{Y}_{1}$ & $\mathrm{Y}_{2}$ & $\begin{array}{c}\text { Catalyst } \\
\text { amount / } \\
\text { mass\% }\end{array}$ & $\begin{array}{c}\text { Total molar } \\
\text { ratio }\end{array}$ & $\begin{array}{c}\text { Conversion / } \\
\%\end{array}$ \\
\hline 1 & -1 & -1 & 5.61 & $11: 1$ & 76.3 \\
2 & -1 & 1 & 5.61 & $17: 1$ & 87.4 \\
3 & 1 & -1 & 10.39 & $11: 1$ & 94.1 \\
4 & 1 & 1 & 10.39 & $17: 1$ & 98.2 \\
5 & -1.41 & 0 & 4.64 & $14: 1$ & 80.7 \\
6 & 1.41 & 0 & 11.36 & $14: 1$ & 98.6 \\
7 & 0 & -1.41 & 8 & $9.77: 1$ & 83.6 \\
8 & 0 & 1.41 & 8 & $18.7: 1$ & 93.9 \\
$9(\mathrm{C})$ & 0 & 0 & 8 & $14: 1$ & 88.3 \\
$10(\mathrm{C})$ & 0 & 0 & 8 & $14: 1$ & 89.8 \\
$11(\mathrm{C})$ & 0 & 0 & 8 & $14: 1$ & 92.3 \\
\hline
\end{tabular}

Coded variables: $\mathrm{Y}_{1}$ (catalyst amount) and $\mathrm{Y}_{2}$ (total molar ratio, TMR). C: central point.

behaviors of both experimental designs were described by a second-order polynomial equation (equation 2).

$$
\mathrm{Y}=\beta_{0}+\sum_{\mathrm{i}=1}^{\mathrm{k}} \beta_{\mathrm{i}} \mathrm{X}_{\mathrm{i}}+\sum_{\mathrm{i}=1}^{\mathrm{k}} \beta_{\mathrm{ii}} \mathrm{X}_{\mathrm{i}}^{2}+\sum \sum_{\mathrm{i}<\mathrm{j}} \beta_{\mathrm{ij}} \mathrm{X}_{\mathrm{i}} \mathrm{X}_{\mathrm{j}}+\varepsilon
$$

where $\mathrm{Y}$ is predicted response (conversion percentage), $\beta_{0}$ is the constant coefficient, $\beta_{\mathrm{i}}, \beta_{\mathrm{ii}}$ and $\beta_{\mathrm{ij}}$ the coefficients for the linear, quadratic and interaction effects, respectively, $X_{\mathrm{i}}$ and $\mathrm{X}_{\mathrm{j}}$ the factors (independent variables) and $\varepsilon$ is the error.

Surfaces were then built using the models for the statistically significant variables. The optimum range of values for the selected variables were obtained by solving 
the regression equation and also by analyzing the response surfaces plots.

\section{Analytical methods}

FAEE quantification was performed by a highperformance liquid chromatography (HPLC) according to the method published by Aryee et al..$^{30}$ Chromatographic analyses were carried out using an HPLC equipment (Shimadzu, model 20AT, Japan) equipped with a quaternary pump, an automatic sampler, a degasser, and a refractive index detector (RID). Chromatographic separations of the compounds were achieved at $40^{\circ} \mathrm{C}$, using a size exclusion column (100 $\AA$ Phenoge $\mathrm{l}^{\mathrm{TM}}$ size exclusion column, $300 \times 7.8 \mathrm{~mm}$ internal diameter, 5-mm, Phenomenex, Torrance, CA, USA). The column was equilibrated and eluted under isocratic conditions using a flow rate of $1.0 \mathrm{~mL} \mathrm{~min} \mathrm{~m}^{-1}$ (mobile phase of $0.25 \% \mathrm{v} / \mathrm{v}$ acetic acid in toluene). The chromatographic run time for each analysis was $17 \mathrm{~min}$. Samples of $100 \mathrm{mg}$ of the fatty system were dissolved in $10 \mathrm{~mL}$ of toluene (HPLC grade) and aliquots of $20 \mu \mathrm{L}$ were injected into the HPLC system. The FAEE samples were quantified by a calibration curve (external calibration), using ten concentration levels of the same pseudocomponents, i.e., acylglycerols in palm olein and ethyl esters. Identification of each pseudocomponent was done in accordance with the retention time of the compound (Figure 2).

\section{Results and Discussion}

\section{Analysis and selection of reaction time}

Figure 3 a shows the FAEE concentration as a function of time, according to the experiments carried out to evaluate the effect of time on the conversion value. The dependence of olein conversion was studied with different amounts of resin (6 and 10 mass $\%$ ) under fixed conditions of MRRM (9:1), and temperature $\left(45^{\circ} \mathrm{C}\right)$. As expected, a higher concentration of FAEE was achieved with an increase in

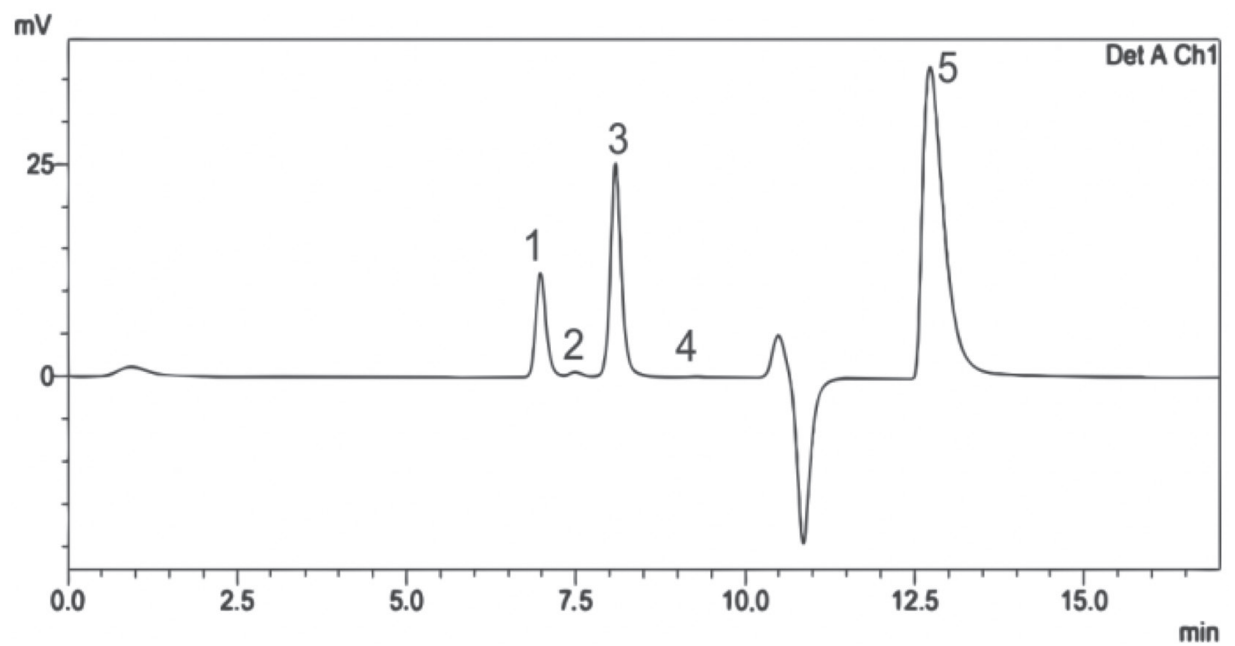

Figure 2. HPLC chromatogram of the transesterification components $(1=$ total triacylglycerol (TAG), $2=$ diacylglycerol (DAG), $3=$ fatty acid ethyl esters (FAEE), $4=$ monoacylglycerols (MAG) and $5=$ ethanol).
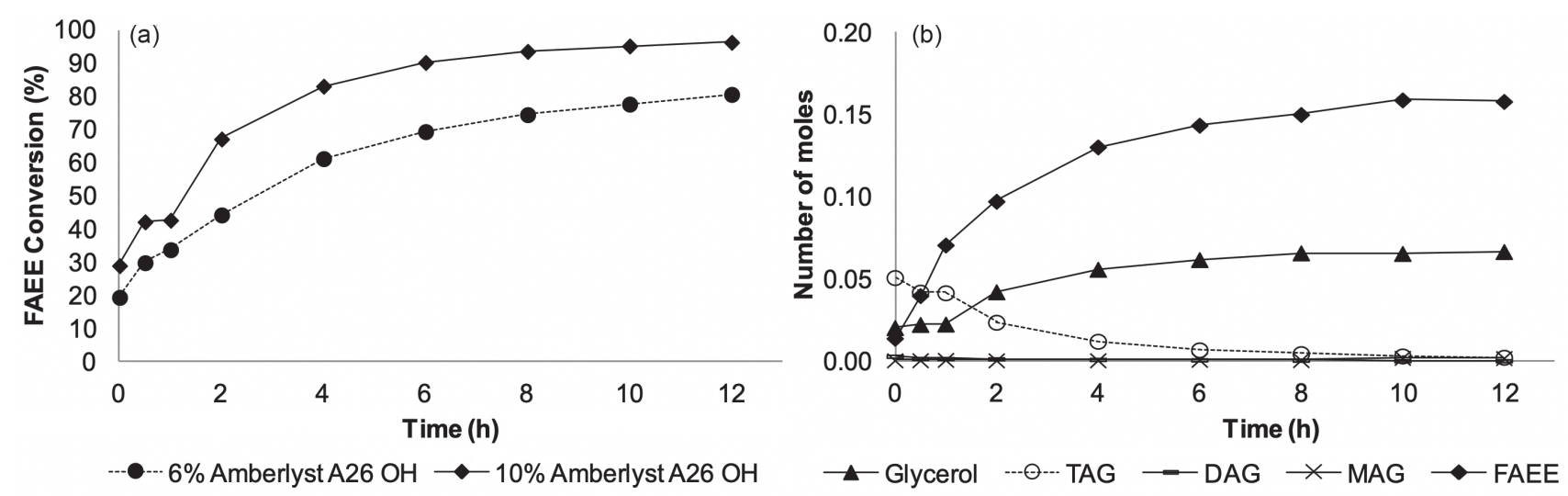

Figure 3. (a) Effect of the reaction time on palm olein transesterification with Amberlyst A26OH resin (6 and 10 mass \%). (b) Number of moles of FAEE, glycerol, tri-, di- and monoacylglycerols as a function of time in the transesterification reaction with 10 mass $\%$ of resin Amberlyst A26OH. 
the quantity of catalyst. The transesterification reaction is catalyzed by the resin active sites, with a larger amount of resin meaning evidently more sites available for olein conversion. After $4 \mathrm{~h}$ of reaction, the conversion value has reached more than $60 \%$ for a catalyst amount corresponding to 10 mass $\%$ of the oil quantity.

The choice of time was based on the conversion after which the reaction speed tends to a very low value. The chosen time was $8 \mathrm{~h}$, a value similar to the literature data obtained using other heterogeneous catalysts. ${ }^{31-33}$ It is important to emphasize that despite its longer reaction time, when compared with the homogeneous catalysts, the raw material with low acidity is not an essential requirement for the heterogeneous catalysis with the Amberlyst A26OH resin. This anion-exchange resin (Amberlyst A26OH) has a good performance in the FFA removal and can be used for the conversion of raw materials with high acidity, as reported by Deboni et al. ${ }^{16}$ This aspect is particularly important in the case of palm oil and its fractions due to their usual high content of FFA.

Figure $3 \mathrm{~b}$ shows the consumption of triacylglycerols and production of FAEE, glycerol, di- and monoacylglycerols over time for the resin content of $10 \%$. As expected, the production of FAEE and glycerol increases and the concentration of TAGs decreases. The concentrations of DAGs and MAGs oscillated over time according to the predominance of their generation or consumption on the three consecutive and reversible reactions of transesterification.

\section{Statistical analysis}

A F-RCCD was applied for analyzing the effects of the MRRM, temperature, and catalyst concentration on the FAEE production (Table 3). The procedure for data analyses was the response surface methodology. The results from the experimental design are presented in Table 3. The maximum acylglyerols conversion to FAEE was $97.0 \%$. The regression model was highly significant, with a coefficient of determination $\mathrm{R}^{2}=0.976$.

Analysis of variance (ANOVA), summarized in Table 5, shows the significance of the linear and quadratic parameters corresponding to the temperature and amount of catalyst ( $p$-value $<0.05$ ) and confirms that the lack of fit was not significant $(>0.05)$.

Equation 3 shows the second order polynomial expression correlating palm olein conversion with the coded independent variables $\left(\mathrm{X}_{1}, \mathrm{X}_{2}, \mathrm{X}_{3}\right)$ :

Conversion $(\%)=89.53+4.04 \times \mathrm{X}_{1}-2.06 \times \mathrm{X}_{1}^{2}+$

$9.29 \times \mathrm{X}_{2}-2.71 \times \mathrm{X}_{2}^{2}+0.15-\mathrm{X}_{3}-0.34 \times \mathrm{X}_{3}^{2}-$

$1.73 \times \mathrm{X}_{1} \times \mathrm{X}_{2}+1.68 \times \mathrm{X}_{1} \times \mathrm{X}_{3}-0.05 \times \mathrm{X}_{2} \times \mathrm{X}_{3}$
Table 5. Analysis of variance (ANOVA) for the polynomial model corresponding to the first experimental design

\begin{tabular}{lccccc}
\hline Factor & $\mathrm{SS}^{\mathrm{a}}$ & $\mathrm{DF}^{\mathrm{b}}$ & $\mathrm{MS}^{\mathrm{c}}$ & $F$-value & $P$-value \\
\hline Model & 1556.2 & 9 & 172.9 & 31.8 & 0.05000 \\
$\mathrm{X}_{1}$ & 223.0 & 1 & 223.0 & 41.0 & 0.00037 \\
$\left(\mathrm{X}_{1}\right)^{2}$ & 47.7 & 1 & 47.7 & 8.8 & 0.02104 \\
$\mathrm{X}_{2}$ & 1177.0 & 1 & 1177.0 & 216.4 & 0.00000 \\
$\left(\mathrm{X}_{2}\right)^{2}$ & 82.9 & 1 & 82.9 & 15.2 & 0.00586 \\
$\mathrm{X}_{3}$ & 0.3 & 1 & 0.3 & 0.1 & 0.82236 \\
$\left(\mathrm{X}_{3}\right)^{2}$ & 1.3 & 1 & 1.3 & 0.2 & 0.63805 \\
$\mathrm{X}_{1}$ by $\mathrm{X}_{2}$ & 23.8 & 1 & 23.8 & 4.4 & 0.07473 \\
$\mathrm{X}_{1}$ by $\mathrm{X}_{3}$ & 22.4 & 1 & 22.4 & 4.1 & 0.08173 \\
$\mathrm{X}_{2}$ by $\mathrm{X}_{3}$ & 0.0 & 1 & 0.0 & 0.0 & 0.95334 \\
Lack of fit & 28.9 & 5 & 5.8 & 1.3 & 0.49689 \\
Residual & 38.1 & 7 & 5.4 & & \\
Pure error & 9.1 & 2 & 4.6 & & \\
\hline Total & 1594.3 & 16 & & & \\
\hline
\end{tabular}

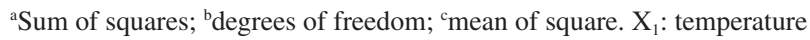
$\left({ }^{\circ} \mathrm{C}\right) ; \mathrm{X}_{2}$ : catalyst amount (mass $\%$ ) and $\mathrm{X}_{3}$ : molar ratio of the mixture, MRRM.

where $X_{1}$ is temperature, $X_{2}$ amount catalyst and $X_{3}$ MRRM.

Figure 4a shows the influence of the MRRM and the catalyst amount on olein conversion at a temperature of $55{ }^{\circ} \mathrm{C}$. It is observed that when the amount of catalyst increases, the conversion increases strongly within the range of MRRM studied. In contrast, the MRRM has a small influence upon the conversion value.

Figure $4 \mathrm{~b}$ indicates the effect of the MRRM and the temperature on the conversion, for a catalyst amount of $11.36 \%$. As shown in this figure, the temperature has a strong influence on the conversion values and this influence is greater for higher MRRM. On the other hand, the MRRM effect shows different behaviors for low and high temperatures. For high temperatures the oil conversion values increase as the molar ratio ethanol:olein (MRRM) increases, a usual behavior observed for reversible reactions such as transesterification. In contrast, at low temperatures the increase of the ethanol amount decreases the conversion values slightly. Contrary to the experience of homogeneous catalysis, the heterogeneous catalysis with a porous catalyst may involve a series of possible controlling steps, for instance: $(i)$ the catalytic activation of the alcoholic reactant that occurs in the resin sites, mostly present within its porous structure, requires the reactant diffusion towards the sites and, (ii) after activation, diffusion outwards to the bulk liquid phase where the reaction probably occurs, (iii) as well as the three reactive stages corresponding to the complete transesterification reaction. This means that the oil conversion performed within a restricted period of time is 

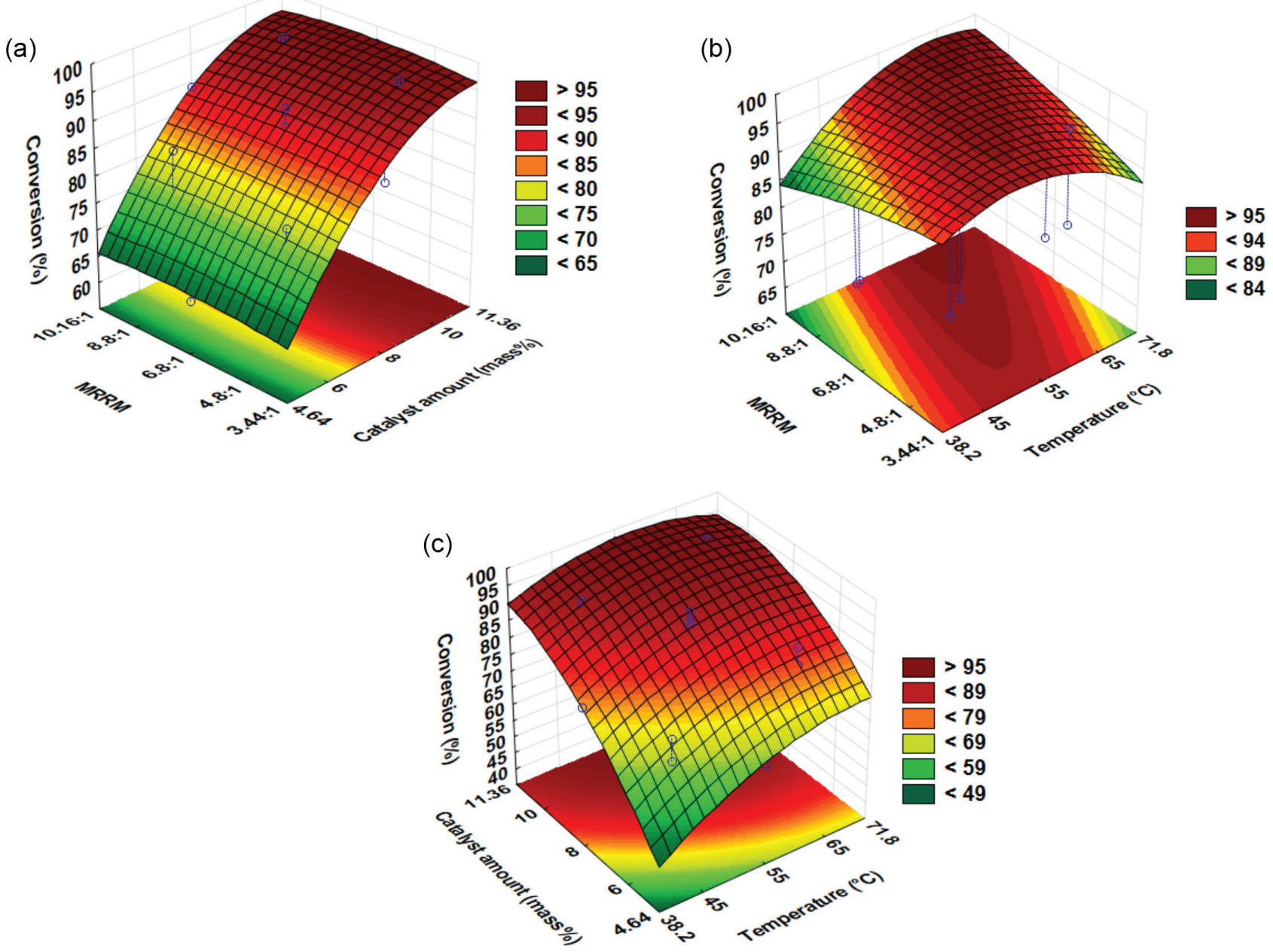

Figure 4. Response surface plots showing (a) interaction between catalyst amount and molar ratio of the reaction mixture (MRRM) at $55^{\circ} \mathrm{C}$; (b) interaction between MRRM and the temperature with $11.36 \%$ of catalyst amount; (c) interaction between catalyst amount and temperature with a MRRM of 6.8:1.

affected by the excess of alcohol as well as by mass transfer and reaction kinetics. At low temperatures mass transfer kinetics may play a more important role mainly due to the liquid phase viscosity inside and around the resin beads. Diffusion is the main mass transfer mechanism within the resin pores and it is highly dependent on liquid viscosity. On the other hand, the stirring of the reaction mixture can overcome, at least in part, the effect of the viscosity increases by improving the convective mass transfer around the resin beads. This means that at low temperatures ethanol diffusion within the solid phase porous structure is slower, affecting the rate of the reactant activation and its transference to the bulk phase. However, a lower ratio ethanol:olein means a higher probability of occurring the alkoxyl nucleophilic attack to the carbonyl groups of the acylglycerol molecules, whose concentration increases due to the lower molar ratio, suggesting the possible predominance of this reaction step at low temperatures.

In Figure $4 c$, the interaction between the catalyst amount and the temperature with a MRRM of $6.8: 1$ is shown. The catalyst amount has a positive influence on the olein conversion within the entire temperature range.

The first experimental design and the corresponding polynomial model suggest an important influence of the temperature upon the conversion values, as shown in Figures $4 \mathrm{a}$ and $4 \mathrm{~b}$. The optimal temperature is close to the central point $\left(55^{\circ} \mathrm{C}\right)$ and in order to reach a maximum conversion to FAEE, a temperature of $56.7{ }^{\circ} \mathrm{C}$ was chosen. Note that this temperature value is close to the maximum operational temperature recommended by the resin datasheet $\left(60^{\circ} \mathrm{C}\right)$. Although in the industrial practice anion resins are sometimes used above this temperature, their operation under stressing temperature conditions accelerates the loss of their active sites.

The results for the second experimental design were obtained at a constant temperature of $56.7{ }^{\circ} \mathrm{C}$ and are presented in Table 4. Note that, in this case, the investigated molar ratio range takes into account the amount of ethanol added to the reaction system together with the catalyst and this additional amount depends on the catalyst quantity. 
The maximum conversion to FAEE was $98.6 \%$. The regression model was highly significant with the coefficient of determination $\mathrm{R}^{2}=0.981$. ANOVA, summarized in Table 6, indicates the significance of the linear parameters of the catalyst concentration and TMR ( $p$-value $<0.05)$. As Table 6 points out, the lack of fit was not significant (>0.05).

Equation 4 shows the polynomial expression correlating olein conversion with the coded independent variables $\left(\mathrm{Y}_{1}, \mathrm{Y}_{2}\right)$ :

Conversion $(\%)=90.13+6.75 \times \mathrm{Y}_{1}-0.29 \times \mathrm{Y}_{1}^{2}+$ $3.73 \times \mathrm{Y}_{2}-0.75 \times \mathrm{Y}_{2}^{2}-1.75 \times \mathrm{Y}_{1} \times \mathrm{Y}_{2}$

where $Y_{1}$ is the amount of catalyst, and $Y_{2}$ is the TMR.

Figure 5 shows the dependence of the olein conversion with the TMR and catalyst amount at a temperature of $57.6{ }^{\circ} \mathrm{C}$. The following behaviors were observed: the conversion increases monotonically for higher catalyst amounts and higher TMR. Furthermore, the results also indicated that higher amounts of catalyst, close to the maximum value of $11.36 \%$, correspond to conversion values less sensitive to the TMR.

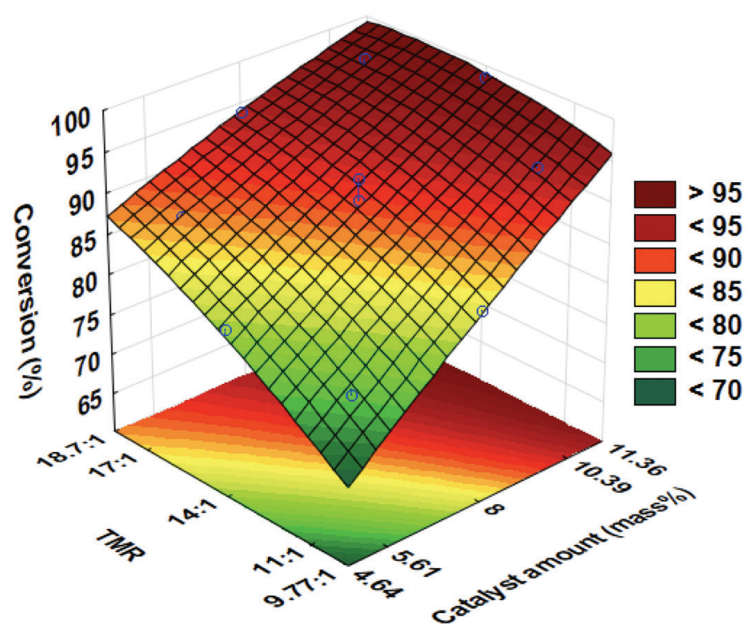

Figure 5. Response surface plot showing interaction between catalyst amount and TMR at $56.7^{\circ} \mathrm{C}$.

In relation to the molar ratio influence both experimental designs seem, in a first evaluation, to show somewhat different results. In fact, the ANOVA analysis for the first experimental design (Table 5) indicates a non-significant influence of the molar ratio, as suggested by Figures $4 \mathrm{a}$ and $4 \mathrm{~b}$. In contrast, the same analysis for the second experimental design (Table 6) shows a significant influence of the molar ratio.

Nevertheless, this difference in the results should be associated with considering or not the amount of ethanol added together with the highly porous catalyst. Firstly, it
Table 6. Analysis of variance (ANOVA) for the polynomial model corresponding to the second experimental design

\begin{tabular}{lccccc}
\hline Factor & $\mathrm{SS}^{\mathrm{a}}$ & $\mathrm{DF}^{\mathrm{b}}$ & $\mathrm{MS}^{\mathrm{c}}$ & $F$-value & $P$-value \\
\hline Model & 489.6 & 5 & 97.9 & 51.2 & 0.05000 \\
$\mathrm{Y}_{1}$ & 363.4 & 1 & 363.4 & 189.9 & 0.00004 \\
$\left(\mathrm{Y}_{1}\right)^{2}$ & 0.5 & 1 & 0.5 & 0.3 & 0.63814 \\
$\mathrm{Y}_{2}$ & 110.8 & 1 & 110.8 & 57.9 & 0.00062 \\
$\left(\mathrm{Y}_{2}\right)^{2}$ & 3.1 & 1 & 3.1 & 1.6 & 0.25843 \\
$\mathrm{Y}_{1}$ by $\mathrm{Y}_{2}$ & 12.3 & 1 & 12.3 & 6.4 & 0.05253 \\
Lack of fit & 1.4 & 3 & 0.5 & 0.1 & 0.94388 \\
Pure error & 8.2 & 2 & 4.1 & & \\
\hline Total & 499.1 & 10 & & & \\
\hline
\end{tabular}

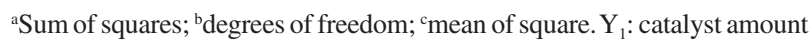
(mass $\%)$ and $\mathrm{Y}_{2}$ : total molar ratio, TMR.

should be noted that the second design covers a range of molar ratios wider than the first one. In this experimental set the TMR varied from 9.77:1 to 18.7:1, values that in terms of MRRM using $11.36 \%$ of catalyst correspond to a range varying from $0.95: 1$ to $9.88: 1$.

It is close to the lowest TMR range, and to the corresponding MRRM values, that conversion decreases in a more significant way. This occurs because the ethanol excess must be provided by the liquid phase available within the resin porous structure. Furthermore, despite some differences concerning the influence of the independent variables, the models derived from both experimental designs indicate similar ranges of optimal operational conditions, as will be shown below.

Both, Pareto analysis (Figure 6) and the obtained models indicate a strong influence of the catalyst amount, in fact, is the variable most important for the first and second statistical designs. Its increase allows very high conversion values, in some cases higher than $98 \%$ or even close to the complete olein conversion. Using the Statistica software and the second polynomial model (equation 4), an almost complete conversion (predicted values close to 99\%) can be obtained with a catalyst amount of $11.36 \%$ and TMR of 14.84:1.

Such a result can also be obtained with other combinations of parameters, for instance with a slightly lower catalyst amount $(10.39 \%)$ and a higher quantity of ethanol (TMR of 18.7:1). Considering the processing steps associated with biodiesel purification and ethanol recycling, it is better to select as operational condition the lower ethanol amount, i.e., a TMR of 14.84:1. In terms of the variable MRRM, that TMR value corresponds to 6.02:1.

On the other hand, a similar procedure performed for the first experimental design indicates high conversion 
(a)

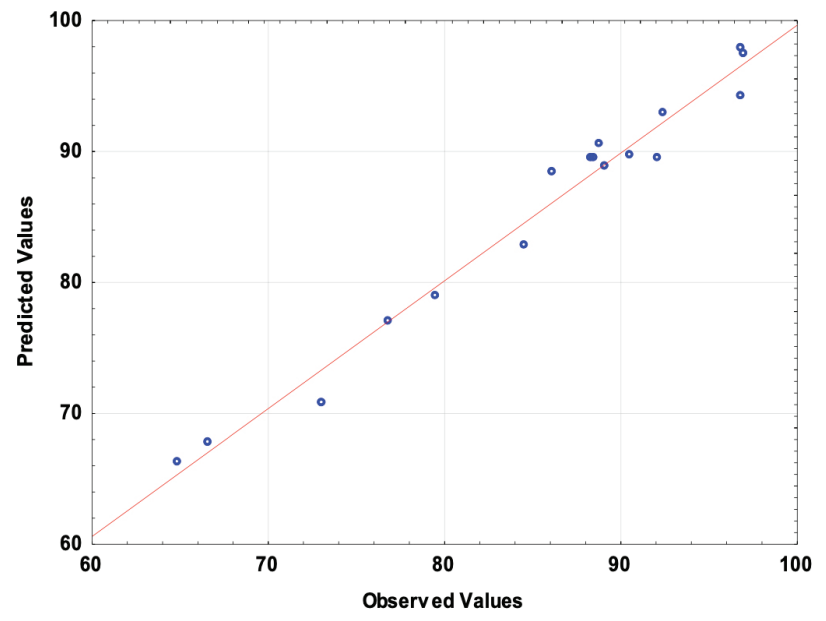

(b)

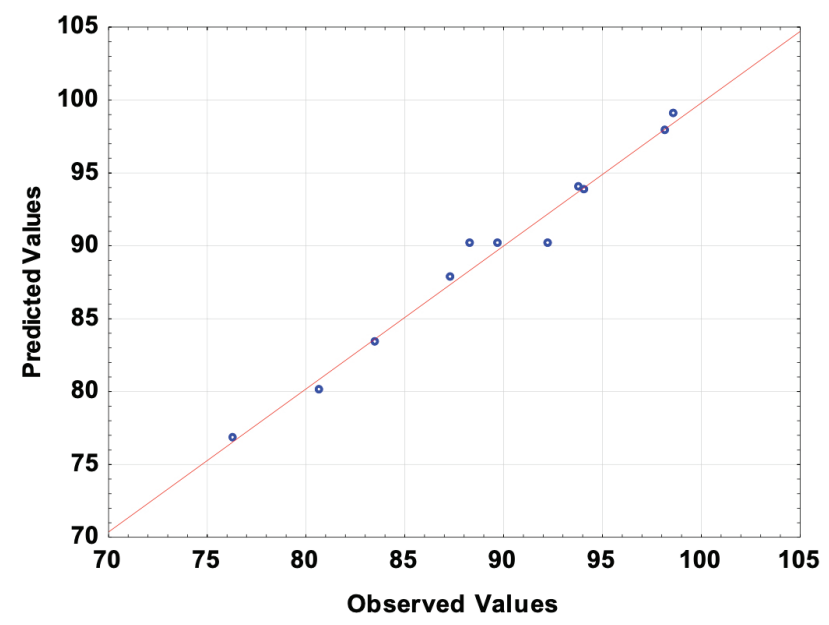

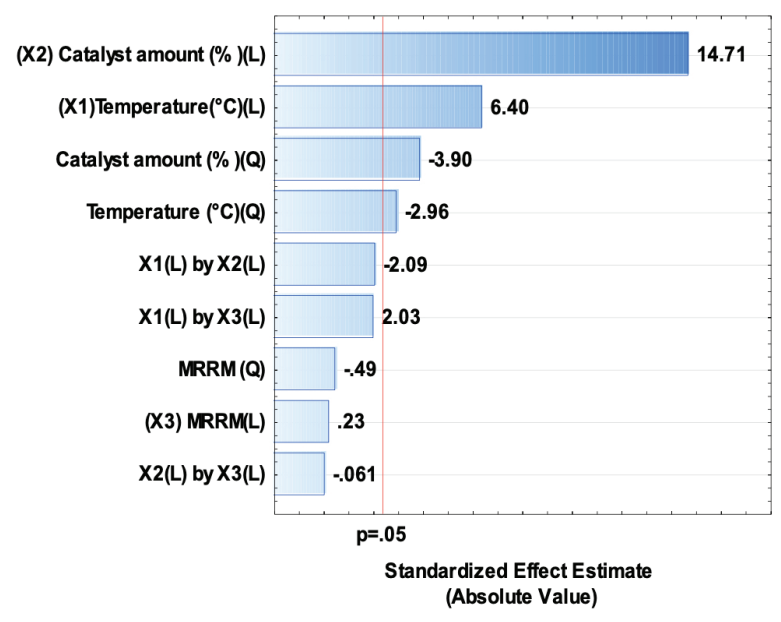

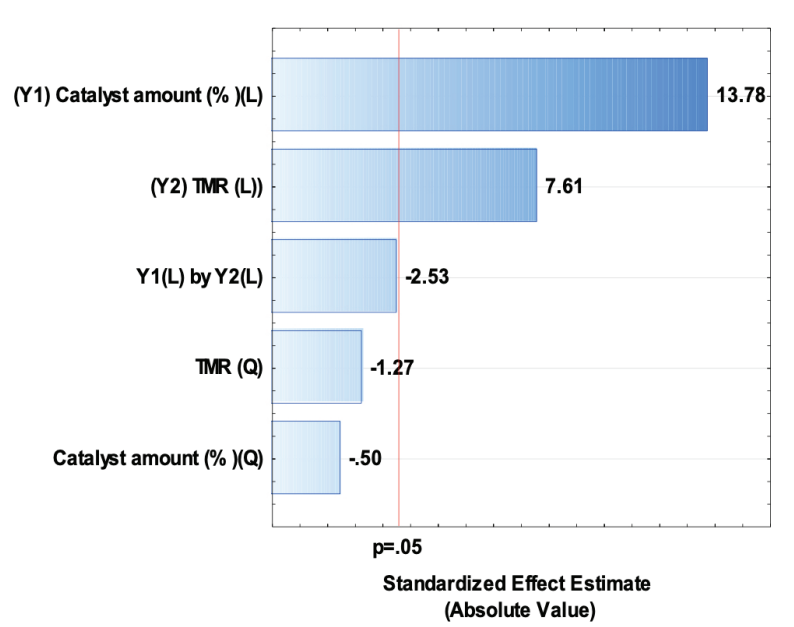

Figure 6. Predicted versus observed plots and Pareto charts for (a) F-RCCD and (b) S-RCCD.

values for the highest amount of catalyst (11.36\%), a result almost independent of the ethanol excess, suggesting the selection of the low excess value (MRRM of 3.44:1). In this case, the reactant value corresponds only to the ethanol amount added to the system as the mixture of reagents, but when one takes into account the amount of ethanol added with the catalyst the corresponding TMR value is $12.26: 1$.

This means that when both molar ratios are expressed on the same basis, they become relatively close. From a technological point of view such a result is quite important. In fact, the proportion of ethanol that should be added to the reactor can be kept within a restricted range from 3.5 to 6.0 times the oil quantity, not much higher than the stoichiometric reaction ratio, and still guarantees a very high conversion. This occurs because the excess of ethanol required for shifting the reaction in the direction of maximal conversion is in part supplied by the ethanol amount steadily present in the catalyst's porous structure. This could reduce costs associated with biodiesel purification and ethanol recycling in a continuous process. As optimal range of operational conditions for guaranteeing conversion higher than $96 \%$ the following values can be suggested: a catalyst amount corresponding to a range from 10.4 to $11.4 \%$ of the oil quantity, a temperature within the range $\left(55\right.$ to $60{ }^{\circ} \mathrm{C}$ ) and a MRRM within the range from 3.5:1 to $6.0: 1$ (or approximately from 12.3:1 to 14.8:1 in terms of TMR).

For the models' validation, experiments were carried out in triplicate in a point within the optimal range of operational conditions: $56.7^{\circ} \mathrm{C}$ of temperature, $11.36 \%$ of catalyst amount for $8 \mathrm{~h}$ and a MRRM of 4.18:1 (equivalent to a TMR of 13:1). Under these conditions the predicted values for the first and second models (equations 3 and 4 ) were 96.62 and $98.63 \%$, respectively. Considering that the standard deviations of the central points for both models are approximately \pm 2 , these predicted values were not significantly different from a statistical point of view. The 
experimental conversion of olein to FAEE was $99.9 \%$, a value higher than those predicted by the models, but still not significantly different from the one calculated according to the second model (equation 4). Anyway, both models seem to be slightly conservative in comparison to the obtained experimental result.

\section{Conclusions}

Ethyl ester production with ethanol using the Amberlyst $\mathrm{A} 26 \mathrm{OH}$ resin was evaluated in order to obtain the optimal range of process parameters by the response surface methodology. The almost complete palm olein conversion to FAEE showed to be viable with the highest amounts of heterogeneous catalyst, temperatures close to the central point and low molar ratios ethanol:oil, under the conditions investigated. The TMR and MRRM presented somewhat different behaviors in the statistical analysis, however similar ranges of optimal values were obtained for both alternatives of expressing the molar ratios of the reagents. The results obtained in the present work are compatible with the mechanism suggested for the transesterification reaction, highlighting the importance of pretreatment for easy accessibility to the resin active sites provided by the alcohol-rich medium available in the solid phase pores. The amount of ethanol carried by the resin within its porous structure makes possible that the quantity of ethanol added to the reactor be not much higher than the required stoichiometric ratio and this still guarantees a very high conversion. The optimal operational conditions, capable of guaranteeing high conversion to ethyl esters (over 96\%) in $8 \mathrm{~h}$, are a catalyst amount corresponding to a range from 10.4 to $11.4 \%$ of the oil quantity, a temperature within the range of 55 to $60^{\circ} \mathrm{C}$ and a MRRM varying from 3.5:1 to $6.0: 1$.

\section{Acknowledgments}

The authors acknowledge CNPq (Conselho Nacional de Desenvolvimento Científico e Tecnológico, 406963/2016-9, 406856/2013-3, 429873/2018-2 and 305870/2014-9), FAPESP (Fundação de Amparo a Pesquisa do Estado de São Paulo, 2014/21252-0 and 2016/10636-8) and CAPES (Coordenação de Aperfeiçoamento de Pessoal de Nível Superior-Brasil, Finance Code 001) for the financial support.

\section{References}

1. Queiroz, A. G.; França, L.; Ponte, M. X.; Biomass Bioenergy 2012, 36, 50 .
2. Yao, K.; Jo-Han, N.; Tung, C.; Shiung, S.; Tong, W.; Renewable Sustainable Energy Rev. 2019, 116, 109399.

3. Demirbas, A.; Energy Convers. Manage. 2008, 49, 2106.

4. Romano, S. D.; Sorichetti, P. A. In Dielectric Spectroscopy in Biodiesel Production and Characterization; Springer: London, 2011, p. 29.

5. Marchetti, J. M.; Miguel, V. U.; Errazu, A. F.; Renewable Sustainable Energy Rev. 2007, 11, 1300.

6. Yusoff, M. F. M.; Xu, X.; Guo, Z.; J. Am. Oil Chem. Soc. 2014, 91, 525.

7. Thoai, D. N.; Tongurai, C.; Prasertsit, K.; Kumar, A.; Fuel Process. Technol. 2017, 168, 97.

8. Esipovich, A. L.; Rogozhin, A. E.; Belousov, A. S.; Kanakov, E. A.; Danov, S. M.; Fuel Process. Technol. 2018, 173, 153.

9. Song, Z.; Subramaniam, B.; Chaudhari, R. V.; Ind. Eng. Chem. Res. 2018, 57, 14977.

10. Fayyazi, E.; Ghobadian, B.; Van de Bovenkamp, H. H.; Najafi, G.; Hosseinzadehsamani, B.; Heeres, H. J.; Yue, J.; Ind. Eng. Chem. Res. 2018, 57, 12742.

11. Shibasaki-Kitakawa, N.; Tsuji, T.; Kubo, M.; Yonemoto, T.; Bioenergy Res. 2011, 4, 287.

12. Veillette, M.; Giroir-Fendler, A.; Faucheux, N.; Heitz, M.; Chem. Eng. J. 2017, 308, 101.

13. Cordeiro, C. S.; da Silva, F. R.; Wypych, F.; Ramos, L. P.; Quim. Nova 2011, 34, 477.

14. Li, J.; Fu, Y. J.; Qu, X. J.; Wang, W.; Luo, M.; Zhao, C. J.; Zu, Y. G.; Bioresour. Technol. 2012, 108, 112.

15. Berk, Z.; Food Process Engineering and Technology: Adsorption and Ion Exchange, $1^{\text {st }}$ ed.; Academic Press: New York, USA, 2009.

16. Deboni, T. M.; Hirata, G. A. M.; Shimamoto, G. G.; Tubino, M.; Meirelles, A. J. A.; Chem. Eng. J. 2018, 333, 686.

17. Ilgen, O.; Akin, A. N.; Boz, N.; Turk. J. Chem. 2009, 33, 289.

18. Tsuji, T.; Kubo, M.; Shibasaki-Kitakawa, N.; Yonemoto, T.; Energy Fuels 2009, 23, 6163.

19. Shibasaki-Kitakawa, N.; Honda, H.; Kuribayashi, H.; Toda, T.; Fukumura, T.; Yonemoto, T.; Bioresour. Technol. 2007, 98, 416.

20. Ren, Y.; He, B.; Yan, F.; Wang, H.; Cheng, Y.; Lin, L.; Feng, Y.; Li, J.; Bioresour. Technol. 2012, 113, 19.

21. Akkarawatkhoosith, N.; Kaewchada, A.; Ngamcharussrivichai, C.; Jaree, A.; BioEnergy Res. 2019, DOI:10.1007/s12155-01910051-4.

22. Eze, V. C.; Harvey, A. P.; Chem. Eng. J. 2018, 347, 41.

23. Rodrigues, K. L. T.; Pasa, V. M. D.; Cren, É. C.; J. Environ. Chem. Eng. 2018, 6, 4531.

24. Datasheet AMBERLYST ${ }^{T M}$ A26 OH Industrial Grade Strongly Basic Polymeric Resin, Dow, 2018, available at https:// www.lenntech.com/Data-sheets/Dow-Amberlyst-A26-L.pdf, accessed in February 2020.

25. Hartman, L.; Lago, R. C.; Lab Pract. 1973, 22, 475. 
26. American Oil Chemists' Society (AOCS); Official Method Ce 1-62: Fatty Acid Composition by Packed Column Gas Chromatography; $4^{\text {th }}$ ed.; Official Methods and Recommended Practices of American Oil Chemist's Society: Champaing, 2009.

27. Antoniosi Filho, N. R.; Mendes, O. L.; Lanças, F. M.; Chromatographia 1995, 40, 557.

28. International Union of Pure and Applied Chemistry (IUPAC); Standard Methods for the Analysis of Oils, Fats and Derivatives, $7^{\text {th }}$ ed.; Blackwell: Oxford, 1987.

29. StatSoft; Statistica v13.1; Dell, USA, 2015.

30. Aryee, A. N. A.; Phillip, L. E.; Cue, R. I.; Simpson, B. K.; Appl. Biochem. Biotechnol. 2011, 165, 155.
31. Abidin, S. Z.; Haigh, K. F.; Sasha, B.; Ind. Eng. Chem. Res. 2012, 51, 14653.

32. Darnoko, D.; Cheryan, M.; J. Am. Oil Chem. Soc. 2000, 12, 1263.

33. Dias, T. P. V. B.; Lunelli, B. H.; Hirata, G. A. M.; Neto, P. M.; Follegatti- Romero, L. A.; Batista, E. A. C.; Meirelles, A. J. A.; Int. J. Eng. Res. Sci. Technol. 2018, 4, 19.

Submitted: October 11, 2019 Published online: February 11, 2020 